\title{
Research on the application of data mining technology in Internet of things
}

\author{
Rui Wang ${ }^{1, a}$, Jinguo Wang ${ }^{2, b^{*}}$, Na Wang ${ }^{3, c}$ \\ ${ }^{1}$ Department of Information Engineering, Jilin Business and Technology College \\ China \\ ${ }^{2}$ Department of Urology, the First Hospital of Jilin University, China \\ ${ }^{3}$ Department of Anaesthesiology, the First Hospital of Jilin University, China

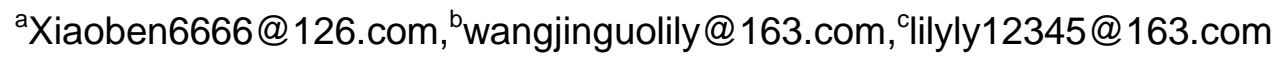 \\ ${ }^{*}$ corresponding author
}

Keywords: data mining, internet of things

\begin{abstract}
For the current Internet of things system architecture analysis, proposed based on the structure of the central node of the structure of the model. The task of center node is that migrate the task of application node to the central node, using intelligent task management program automatically complete the task. One of the basic tasks of the central node is a routine report, the main content is to submit a judgment of normal data. Research on series of internet of thing's data, given an abnormal sequence detection scheme. In this paper, we use the KNN method in data mining to realize the detection of abnormal sequences, and the automatic detection of anomaly detection is realized by the artificial judgment anomaly to the density deviation on the algorithm.
\end{abstract}

\section{Introduction}

The essence of data mining is the process of finding regular patterns from a large number of complex data, so data mining can be applied in every field of life and work. As a popular subject, its scientific theory is becoming more and more perfect, and it is needed to apply these theories to the practical life to produce effective social and economic value. In this paper, the application of data mining in the Internet of things as the breakthrough, through the use of data mining technology to solve specific problems.

The Internet of things is a kind of network application, which is based on the network construction to realize the mutual relation between the object and the object. Applications of the Internet of things technology include: intelligent sensing, machine learning, pattern recognition as well as network pervasive computing and network integration. The characteristics of the Internet of things are the realization of the information and communication between people and things, or the exchange of information between people and objects by constructing the local network. The Internet of things as a new field, combined with the current sensing technology and network technology will greatly improve the way people live. It is essential to establish the information processing system of the Internet of things for playing the great potential of the Internet of things. It is a difficult problem that how to management information processing system automation and intelligently in the development of the Internet of things. 


\section{Design of the central node of the Internet of things}

Internet of things as the next important scientific development proposition, but there are still a lot of problems in its application. There are problems of technical, standard specification and product development. There are also a variety of issues in the overall planning. These problems include industrial planning, institutional mechanisms, collaborative cooperation, promotion and application, etc..

According to the different types of sensors can be divided into sensing detector and control detector. Sensing detector is used to perceive the surrounding environment and transmission through the network will transmit real-time data to the application layer, function and structure of this type of detectors are generally relatively simple only the signal transmitting device no command receiving device, the main task is to monitor specific indicators for specific tasks, and is generally used in the feedback signal of periodic independent power supply system. The control detector is a detector that has a controllable unit, can accept the signal and respond in time, can receive the specific control signal and respond to the signal. Because the sensor types on the market vary. In addition, with the development of the Internet of things and the construction of a variety of things, the coexistence of a variety of detectors is more and more popular, if only the use of ordinary signal transmission and reception system is difficult to meet the demand. This requires a responsible for the transfer of the smart module for receiving and forwarding instructions of the task, the module is the central node.

For the application layer, setting a center node can automatically handle daily monitoring tasks only special situations and daily reports as a result of terminal transfer to specific functions can greatly simplify the application terminal operation. In the center of the node deployment procedures used to identify normal and non normal situation, the deployment of format conversion program to achieve the standardization of data cache system deployment for the original data stored for a period of time in the cache and uploaded to the original database. The identification of the state as well as the application layer of the information needed to transfer the property.

\section{The introduction of the center node in Internet of things}

Data reception. First of all, determining the use of the Internet of things, the purpose is to know the scope of the use and to determine the use of which type of sensor. The center node is determined to be able to sense all of the sensors, while allowing the central node to extend the type and number of sensors. We can deploy the wireless receiving device to the center node, and the sensor can detect the signal in a certain period and send it to the center node through the ZigBee wireless network after the sensor is converted into the data information.

Data cache. Fixed time in the central node will cache a certain data, the purpose is to be used for intelligent analysis. The cache data of the central node will be uploaded to the original database regularly, and the results will be given back to the application layer after the intelligent analysis is completed.

pattern recognition. If all the data is transmitted to the application control platform, the calculation of the data will not be able to play the advantages of the distributed center node, which can be used to generate the normal data mining model. Once the processed data for pattern matching is normal. This is the result of the tag and the processed data to the application layer. 
Access control. Different applications can obtain different data, such as: data warehouse can get the original data center node. The application terminal can obtain the pattern report; when the center node is set up, we can also open some original data items.

Multiplexed sensor node. Getting the access code of the sensor to set up a central node can realize the reuse of the sensor. This can be a flexible use of limited sensing resources to deploy more central nodes to complete the extension of multi application tasks.

Adaptive center node. In the monitoring network application, a very important function of the central node is to realize the self adaptation of the model algorithm. The main reason is that the data detected by the local small environment is very different. And when the Internet of things to reach a certain scale, all rely on the cost of manual data analysis will be very expensive. Therefore, the central node to complete a certain amount of data processing work here is the daily report, and according to the environment to change the dynamic adjustment will greatly improve the efficiency of the Internet of things.

\section{Conclusion}

Data mining will become the future development direction of the Internet of things. From the practical application of data mining as a large amount of data, fuzzy, extract a potential useful information among them, not only to deepen the application of the Internet of things, also provides a broad prospect out of the homogenization of competition for enterprises. In the long run, data mining will become the development direction of the entire industry.

\section{References}

[1] Lei Shi,Lei Xi,Xinming Ma,Mei Weng,Xiaohong Hu. A novel ensemble algorithm for biomedical classification based on Ant Colony Optimization[J]. Applied Soft Computing Journal . 2011 (8)

[2] S.K. Mathanker,P.R. Weckler,T.J. Bowser,N. Wang,N.O. Maness. AdaBoost classifiers for pecan defect classification[J]. Computers and Electronics in Agriculture . 2011 (1)

[3] Lei Shi,Xinming Ma,Lei Xi, Qiguo Duan,Jingying Zhao. Rough set and ensemble learning based semi-supervised algorithm for text classification[J]. Expert Systems With Applications . $2010(5)$

[4] Changseok Bae,Wei-Chang Yeh,Yuk Ying Chung,Sin-Long Liu. Feature selection with Intelligent Dynamic Swarm and Rough Set[J]. Expert Systems With Applications . 2010 (10)

[5] Broll, Gregor,Rukzio, Enrico,Paolucci, Massimo,Wagner, Matthias,Schmidt, Albrecht,Hussmann, Heinrich. Perci: Pervasive Service Interaction with the Internet of Things[J]. IEEE Internet Computing . 2009 (6)

[6] Ramiz M. Aliguliyev. Performance evaluation of density-based clustering methods[J]. Information Sciences . 2009 (20)

[7] C. Kamphuis,H. Mollenhorst,A. Feelders,D. Pietersma,H. Hogeveen. Decision-tree induction to detect clinical mastitis with automatic milking[J]. Computers and Electronics in Agriculture . 2009 (1)

[8] Ashish Ghosh,Anindya Halder,Megha Kothari,Susmita Ghosh. Aggregation pheromone density based data clustering[J]. Information Sciences . 2008 (13)

[9] Maurizio Filippone,Francesco Camastra,Francesco Masulli,Stefano Rovetta. A survey of kernel and spectral methods for clustering[J]. Pattern Recognition . 2007 (1) 
[10] Masahiro Inuiguchi,Takuya Miyajima. Rough set based rule induction from two decision tables[J]. European Journal of Operational Research . 2006 (3)

[11] J. Bazan,H. S. Nguyen,P. Synak,J. Wroblewski."Rough set algorithms in classification problem,". Rough Set Methods and Applications . 2000

[12] Hansen L.K,Lusberg L,Salamon P.Ensemble methods for handwritten digit recognition. Proceedings of the IEEE Workshop on Neural Networks for Signal Processing . 1992

[13] Zhang Y,Meratnia,Havinga.Adaptive and Online One-Class Support VectorMachine-Based Outlier Detection Techniques for Wireless SensorNetworks. Advanced Information Networking and ApplicationsWorkshops . 2009

[14] Rajasegarar S,Leckie C,Palaniswami M,et al.Quarter sphere based distributed anomaly detection in wireless sensor networks. IEEE International Conference on Communications . 2007 